\title{
Farey sequences of spatiotemporal patterns in video feedback
}

\author{
B. Essevaz-Roulet, ${ }^{1}$ P. Petitjeans, ${ }^{1}$ M. Rosen, ${ }^{2}$ and J. E. Wesfreid ${ }^{1}$ \\ ${ }^{1}$ Laboratoire de Physique et Mécanique des Milieux Hétérogènes, CNRS UMR 7636, École Supérieure de Physique et Chimie \\ Industrielles, 10 rue Vauquelin, 75231 Paris Cedex 05, France \\ ${ }^{2}$ Grupo de Medios Porosos Facultad de Ingenieria, Universidad de Buenos Aires, Paseo Colon 850, 1063 Buenos Aires, Argentina
}

(Received 15 June 1999; revised manuscript received 15 October 1999)

\begin{abstract}
In this paper we present an experimental and theoretical description of the dynamic of spatial patterns obtained in a video feedback loop. A video camera monitors the screen to which it is connected and can turn around its optical axis at an angle $\alpha$. Under certain conditions of brightness and magnification, this optoelectronic system produces spatiotemporal patterns in the form of spots located on a circle on the screen. These patterns are very similar to the spatial transverse modes obtained in other optical devices such as lasers or photorefractive media. It is possible to generate stationary patterns of $n$-fold symmetries for angles $\alpha$ $=2 \pi / n$. When the angle $\alpha$ varies around $2 \pi / n$, the pattern rotates with a certain frequency proportional to the difference between $2 \pi / n$ and $\alpha$. We discover more general patterns at angles $2 \pi /(p / k)$ with $p$-fold symmetry, following the hierarchy of the Farey algorithm which theoretically can produce stationary patterns at any angle $\alpha$. Very accurate experiments were performed to observe these patterns up to the level $k=6$. This is the first time a Farey tree has been observed as a sequence of spatial patterns to our knowledge. Previous observations of this hierarchy were made only in the temporal domain.
\end{abstract}

PACS number(s): 42.65.Sf, 45.70.Qj, 47.20.Ky, 47.54.+r

\section{INTRODUCTION}

Physical systems where the "input" receives phase or amplitude information from the "output," inducing a delayed feedback loop, can show interesting temporal behavior, including self-oscillations. Regular and chaotic spatiotemporal regimes have been obtained in different systems with these characteristics, arising from electronics circuits [1], hydrodynamic self-oscillators [2], optics [3-11], and laser devices [12]. A particular example of hydrodynamic nature is given by the jet instability: the shear layer instability of a free jet is convectively unstable (no noise amplification, showing a large temporal spectrum). When an obstacle is put in its path, as in the case of the geometry called "jet edge" or the "edge tone," the spectrum shows a well-defined peak, as in the case of absolute instabilities. This global coherent behavior is due to the feedback introduced between the output and the input, by any kind of temporal delayed physical information of acoustic or hydrodynamic nature. In laser physics, present interest is focused on the dynamics of low frequency bursts produced by reinjection of the output signal into the cavity, especially in the case of semiconductor lasers [12]. In spatially extended systems, the feedback and coupling of oscillators can generate particular modes including ordered patterns. In general, the coupling mechanism (diffusive or nonlocal, with specified long range correlation) and nonlinear saturation allow for the emergence of particular patterns. Experiments with the nonlinear photorefractive index, such as those with liquid crystals, where light is reinjected and where, in addition, the image is shifted, generate spatial patterns that are the most similar to those obtained in the experiments described in this paper [6]. This problem including a feedback loop is an active subject of research in the field of nonlinear dynamical systems, in order to understand the complex behavior of turbulence (especially spatiotemporal intermittency) as well as study living systems such as neural networks and cardiac waves. This paper pre- sents an experiment that allows for the study of spatiotemporal patterns produced with a delayed feedback loop where most of the parameters can be controlled: the video feedback system. Video feedback [13] is an optical loop produced when a video camera monitors the screen to which it is connected. The camera can rotate around its optical axis at an angle $\alpha$ with respect to the monitor.

This optoelectronic arrangement can produce peculiar spatiotemporal patterns with interesting symmetries and was experimentally studied by Hausler and co-workers [14-17], Crutchfield [18], and Goulobev et al. [19]. The existence of these patterns can be explained on the basis of the feedback loop: the input (video camera) is nonlocally coupled to the angular-shifted output (monitor), which sends information to the input, and so on. The saturation in the pixel intensity in the photosensitive sensor is the source of nonlinearity. In addition, short range coupling is obtained by "diffusion" between an excited pixel and its nearest neighbors on the sensor, so that they are stimulated even if only one pixel was first excited. This question has been studied in Refs. [14$18]$. Very accurate experiments on the spatiotemporal evolution of the patterns observed in this system are presented in this paper. An intermediate state is pointed out that follows the Farey scenario [20-23] in the spatial symmetries generated by this optoelectronic device.

\section{EXPERIMENTAL SETUP}

The "feedback loop" is obtained by making the camera watch the center of the monitor to which it is directly connected (Fig. 1). The camera is a classical charge-coupled device black and white video device. It can turn around its optical axis with an angle $\alpha$ thanks to a microcontrol stage which allows an angle accuracy of 1 minute. This is much better than what was achieved in most previous experiments on this subject. This leads to a relative accuracy of order $10^{-4}$ in the case of $n=4, \alpha=2 \pi / 4$ (see Sec. III for the 


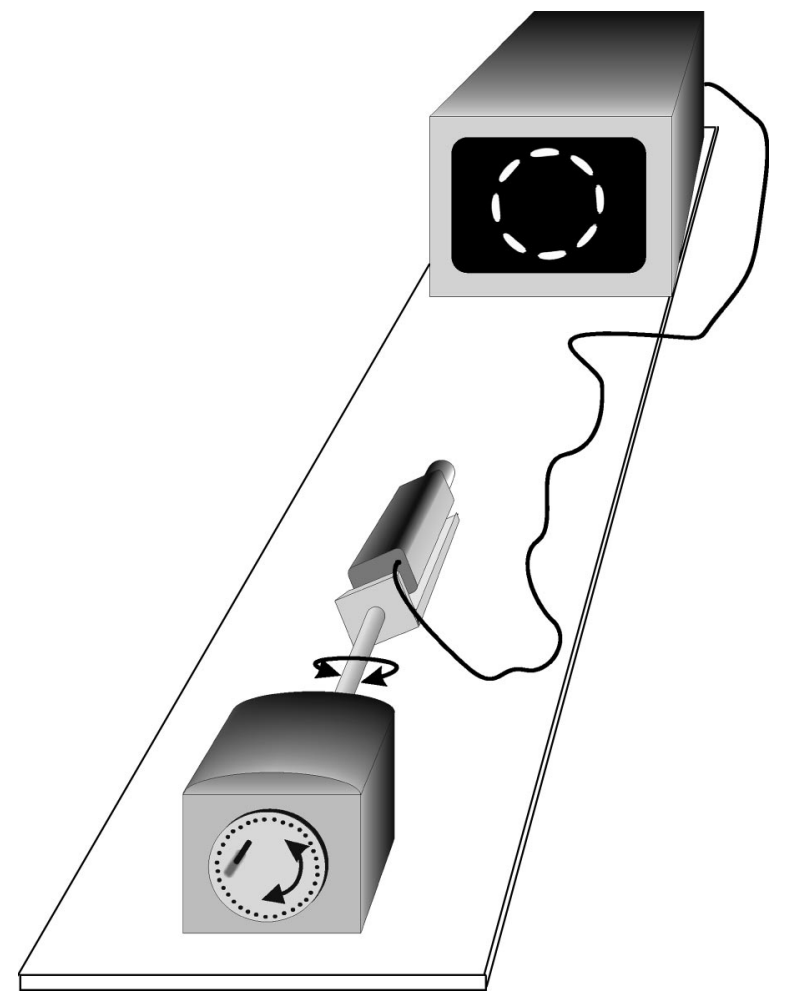

FIG. 1. Experimental setup.

pattern description). Different pattern configurations can appear depending on the parameters of this optoelectronic system. The main parameters are the magnification, the focus and diaphragm aperture for the camera, and the brightness and contrast for the monitor. If the camera is defocused from the image on the screen, a diffusionlike effect is produced. Indeed, defocusing allows for the information of each pixel to be received by the surrounding pixels. The characteristic diffusion length is scaled by the gap between the focused and defocused positions. In our experiments, those parameters are chosen so as to get clear images and low diffusion. The ambient light is chosen to be dark. When the magnification ratio $R$ is smaller than 1 , the image is reduced at each loop until the original image is lost. If the angle $\alpha$ is not zero, the image is rotated with respect to the previous one, resulting in an image with a spiral aspect. This pattern is static since each feedback loop induces exactly the same process [Fig. 2(a)]. In contrast, when the magnification is larger than 1, every image of the central portion of the monitor grows to the dimensions of the screen. When $\alpha$ is not zero, the observed pattern looks like a sudden spiral burst with irregular frequency [Fig. 2(b)]. Under certain conditions, we obtain the equivalent of optical developed turbulence. In this paper, we

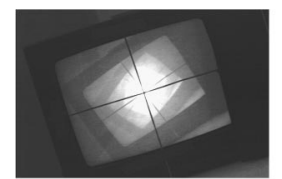

$\mathrm{R}<1$

(a)

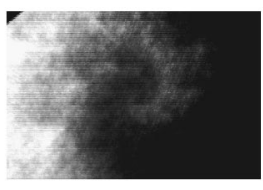

$\mathrm{R}>1$

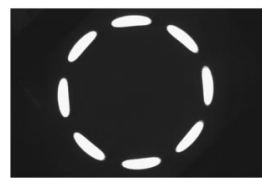

$\mathrm{R}=1$
FIG. 2. Three typical patterns obtained by video feedback. $R$ is the magnification ratio.
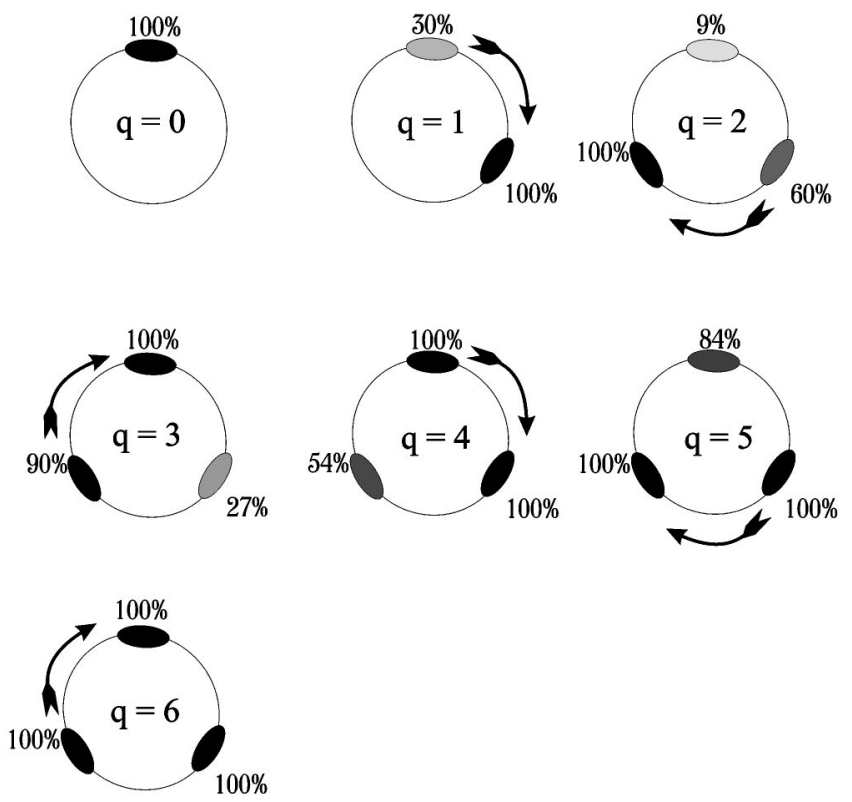

FIG. 3. An initial perturbation (here directly saturated light, shorter than the loop duration) propagates on the screen and $2 n$ feedback loops are needed to build an $n$-spot pattern. The case $n$ $=3$ is shown here with six loops until the three spots are saturated (gray levels are inverted), with persistence $P=30 \%$. At each loop, one spot has intensity equal to the sum of $30 \%$ of the same spot intensity in the previous loop, and the total intensity of the spot situated just before it in the previous loop.

are interested in the case where there is no magnification (the ratio $R$ is equal to unity) [Fig. 2(c)].

\section{PATTERN DESCRIPTION}

When there is no magnification, the image of the screen has the same size as the original one. If $\alpha=0$, the image is superimposed on the previous one and a large white spot is observed.

The persistence (remaining intensity) of the camera is $30 \%$ after $40 \mathrm{~ms}$ (time of one feedback iteration). The persistence of the screen is $5 \mathrm{~ms}(0 \%$ remains after $40 \mathrm{~ms})$ and the delay of the electronic setup is negligible. In consequence, the resulting image saturates if the light intensity is enhanced because each image is superimposed on the remainder of the preceding one. In contrast, the resulting image vanishes if the light intensity of an image is less than the previous one. In the experiment, the parameters are chosen such that white patterns are observed on a dark background. Under these conditions white spots located regularly on a circle are observed [Fig. 2(c)]. This pattern can be stationary or not (rotation), as explained later (Sec. V).

When $\alpha=2 \pi / n$ (where $n$ is an integer), the feedback loops create an $n$-fold symmetry, leading to $n$ stationary spots located on a circle [Fig. 2(c)]. Hence, when $\alpha$ is exactly $2 \pi / n$, the pattern is rotated at each loop by $2 \pi / n$ rad, and exactly matches the remainder of the previous one, giving a stationary image. Figure 3 describes the transient process during which the $n$ spots are built. An adequate initial perturbation (light with intensity equal to or larger than what is needed to reach a white spot) is needed to initiate the processes. If the perturbation is shorter than the duration of 
TABLE I. Example of the intensity $X_{n}(t=q \tau, i) / X_{0}$ at time $t=q \tau$ in the case $n=3$. The intensity at time $t=q \tau$ is the sum of the intensities of the spot $i-1$ and the persistence of the spot $i$ at the previous loop [time $(q-1) \tau$ ]. The sum of the intensities of all spots at a given time $t=q \tau$ is $(P+1)^{q} X_{0}$.

\begin{tabular}{llllllllll}
\hline \hline & $t=0$ & $t=\tau$ & $t=2 \tau$ & $t=3 \tau$ & $t=4 \tau$ & $t=5 \tau$ & $t=6 \tau$ & $t=7 \tau$ & $t=8 \tau$ \\
\hline$i=1$ & 1 & $P$ & $P^{2}$ & $P^{3}+1$ & $P^{4}+4 P$ & $P^{5}+10 P^{2}$ & $P^{6}+20 P^{3}+1$ & $P^{7}+35 P^{4}+7 P$ & $P^{8}+56 P^{5}+28 P^{2}$ \\
$i=2$ & 0 & 1 & $2 P$ & $3 P^{2}$ & $4 P^{3}+1$ & $5 P^{4}+5 P$ & $6 P^{5}+15 P^{2}$ & $7 P^{6}+35 P^{3}+1$ & $8 P^{7}+70 P^{4}+8 P$ \\
$i=3$ & 0 & 0 & 1 & $3 P$ & $6 P^{2}$ & $10 P^{3}+1$ & $15 P^{4}+6 P$ & $21 P^{5}+21 P^{2}$ & $28 P^{6}+56 P^{3}+1$ \\
Total & $(P+1)^{0}$ & $(P+1)^{1}$ & $(P+1)^{2}$ & $(P+1)^{3}$ & $(P+1)^{4}$ & $(P+1)^{5}$ & $(P+1)^{6}$ & $(P+1)^{7}$ & $(P+1)^{8}$ \\
\hline \hline
\end{tabular}

one feedback loop, a certain number of loops is needed to obtain a permanent image (see Fig. 3). If the perturbation remains in its position for a longer time, then only $n$ loops are needed to generate the whole pattern. This longer time of perturbation should be at least the time needed for the $n$ loops.
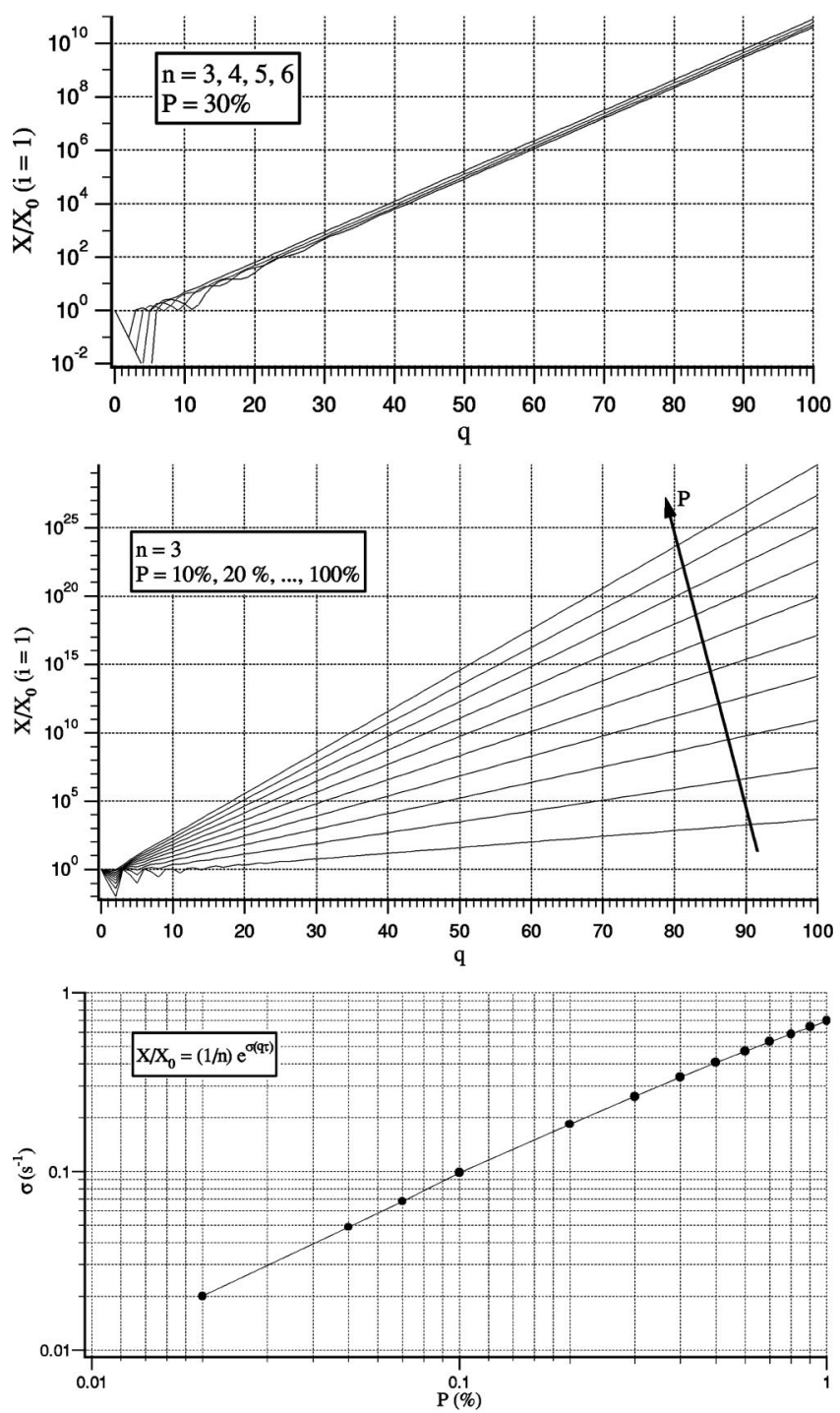

FIG. 4. (a) Intensity $X / X_{0}$ of the spot number $i=1$ as a function of the iteration number $q$ for the cases $n=3,4,5$, and 6 spots, and for a persistence $P=30 \%$. (b) Intensity $X / X_{0}$ of the spot number $i$ $=1$ as a function of the iteration number $q$ for the case $n=3$, and for different persistence $P$. (c) Growth rate $\sigma$ as a function of the persistence $P$.
It is possible to express the intensity $X$ of each spot at the location $i$ at any time $t$ as a function of the initial perturbation intensity $X_{0}$, the number $n$ of spots, and the persistence $P$ after one loop. An example of the intensity $X_{n}(t$ $=q \tau, i) / X_{0}$ at a time $t=q \tau$, i.e., after $q$ iterations (or feedback loops), is given in Table I in the case $n=3$. The intensity at time $t=q \tau$ is the sum of the intensities of the spot $i$ -1 and the persistence of the spot $i$ from the previous loop [time $(q-1) \tau]$.

The sum of the intensities of all spots at a given time $t$ $=q \tau$ is $(P+1)^{q} X_{0}$. From this observation, an expression for the intensity of each spot has been deduced and is given by

$$
X_{n}(t=q \tau, i)=\sum_{j=0}^{n j+i-1 \leqslant q} C_{q}^{n j+i-1} P^{q-n j-i+1} X_{0} .
$$

The parameter $P$ takes the value of zero when there is no persistence and 1 when the persistence is total. The intensity globally increases with time as shown Fig. 4(a). The slope of the curve does not depend on the number of spots $n$ (the angle is $\alpha=2 \pi / n)$, but the time needed to reach a quasipermanent growth of the intensity is longer if the number of spots increases.

The growth of the intensity with time depends on the persistence $P$ with a quasiexponential law as shown in Fig. 4(b). The analytical expression is given by the formula (1), which can be expressed with hypergeometric functions. In the case $n=3$, the hypergeometric function reduces to the expression

$$
\begin{aligned}
\frac{X_{n=3}(t=q \tau, i=1)}{X_{0}}= & \frac{1}{3}\left[\left(1+\frac{1}{P}\right)^{q}+\left(1-(-1)^{1 / 3} \frac{1}{P}\right)^{q}\right. \\
& \left.+\left(1+(-1)^{2 / 3} \frac{1}{P}\right)^{q}\right] P^{1 / 3}
\end{aligned}
$$

This law tends asymptotically to an exponential growth:

$$
\frac{X}{X_{0}}=\frac{1}{n} e^{\sigma t}
$$

where $t=q \tau$. The growth rate $\sigma$ is plotted as a function of $P$ in Fig. 4(c), and shows an evolution like $\sigma \sim P^{0.9}$. Whatever the initial perturbation intensity, the maximum intensity reached by the system cannot exceed a saturation value $X_{\max }$ imposed by the system itself. In that case, the intensity increases exponentially until the limiting value $X_{\max }$, above which the system cannot go. This is a linear description since the intensity is built as the sum of the intensities of the spot $i-1$ and the persistence of the spot $i$ from the previous loop 
[time $(q-1) \tau]$. The equation governing the temporal evolution of the intensity $X$ can be written as follows:

$$
\frac{d X}{d t}=K X,
$$

where $K$ is a constant, and where Eq. (3) is the solution. This equation is similar to the linear amplitude equation of classical linear stability problems. It is possible to imagine physical systems where the persistence $P$ would depend on the intensity $X$, leading to more complex behaviors: $P$ $=P(X)$. Then, the system can reach its maximum value $X_{\max }$ with a different law. This implies nonlinearity, which would depend on $P(X)$.

A new important behavior to note is that the system can reach another equilibrium state by disturbing the basic pattern. This disturbance is created by occulting a small part of the screen during a short time. This other pattern, stationary or not, does not obey the law $n=2 \pi / \alpha$, i.e., it has a number of spots different from $n$ or $n+1$ although the angle $\alpha$ is between $2 \pi / n$ and $2 \pi / n+1$. This complex feature can be understood with the help of the Farey tree.

\section{FAREY TREE}

The Farey tree is a geometrical representation of a numerical series. Between two consecutive integers $n$ and $n$ +1 , all the nonirrational numbers are given by the ratio $c / k$. Those ratios are represented on a two dimensional diagram, where $k$, called the "level," is on the ordinate axis. The ratios $c / k$ are located on the abscissa with their numerical value. The general relation to build a Farey tree is as follows:

$$
\frac{c}{d}=\frac{k a+a^{\prime}}{k b+b^{\prime}},
$$

with $a b-c d=1$, and where the coefficients are related to preceding nodes (see Ref. [24] for more details). In the case of two coupled oscillators [20], the series is reduced as fol- lows (Fig. 5). From the two parents $a_{1}$ at the level $k_{1}$ and $a_{2}$ at the level $k_{2}$ the new number $c$ at the level $k$ is given by the relation

$$
c=a_{1}+a_{2}, \quad k=k_{1}+k_{2} .
$$

In our case $c, a_{1}$, and $a_{2}$ correspond to the number of spots. At the level $k=1$, the number $a_{1}$ is equal to $n$, and $a_{2}$ is equal to $n+1$, as described before. As already suggested, stationary patterns were observed for angle $\alpha$ between $2 \pi / n$ and $2 \pi /(n+1)$. The Farey tree representation shows that for any rational angles $\alpha$ between $2 \pi / n$ and $2 \pi / n+1$, a stationary pattern exists with a number of spots different from $n$ or $(n+1)$. The case $\alpha=2 \pi / n$ appears to be the particular case $k=1$, and the other patterns correspond to $k>1$. For each new pattern $(k>1)$, its number of spots $c$ results from the summation of the numbers of spots $a_{1}$ and $a_{2}$ at lower levels $k_{1}$ and $k_{2}$ as shown in Eq. (6). The number $c$ of stationary spots at a level $k$ is given by $k / c=\alpha / 2 \pi$, where $k / c$ is the irreducible ratio of $\alpha / 2 \pi$. In the experiment, stationary spots up to level $k=6$ were observed. For example, 19 stationary spots were obtained for $\alpha=1.98 \mathrm{rad}=6 / 19 \times 2 \pi$ (Fig. 5).

The level $k$ is understood as the number of revolutions needed to build $c$ spots (Fig. 6). For instance, nine spots can be built by adding one spot just after the previous one, i.e., the first one at location 1 , the second at location 2, the third at 3 , corresponding to the level $k=1$ at the angle $\alpha$ $=1 / 9 \times 2 \pi$ [Fig. 6(a)]. However, nine spots can also be built by adding one spot by jumping over one location, i.e., the first spot on the first location, but the second one on the third location, the third one on the fifth location, and so on, in such a way that the final pattern is built after exploring the complete circle twice, which corresponds to the level $k=2$ at the angle $\alpha=2 / 9 \times 2 \pi$ [Fig. 6(b)].

Equation (1) for the intensity of the spots can be generalized to any level $k$. The intensity $X_{c, k}(t=q \tau, i)$ of the spot $i$ at the time $t=q \tau$, i.e., after $q$ iterations (or feedback loops),

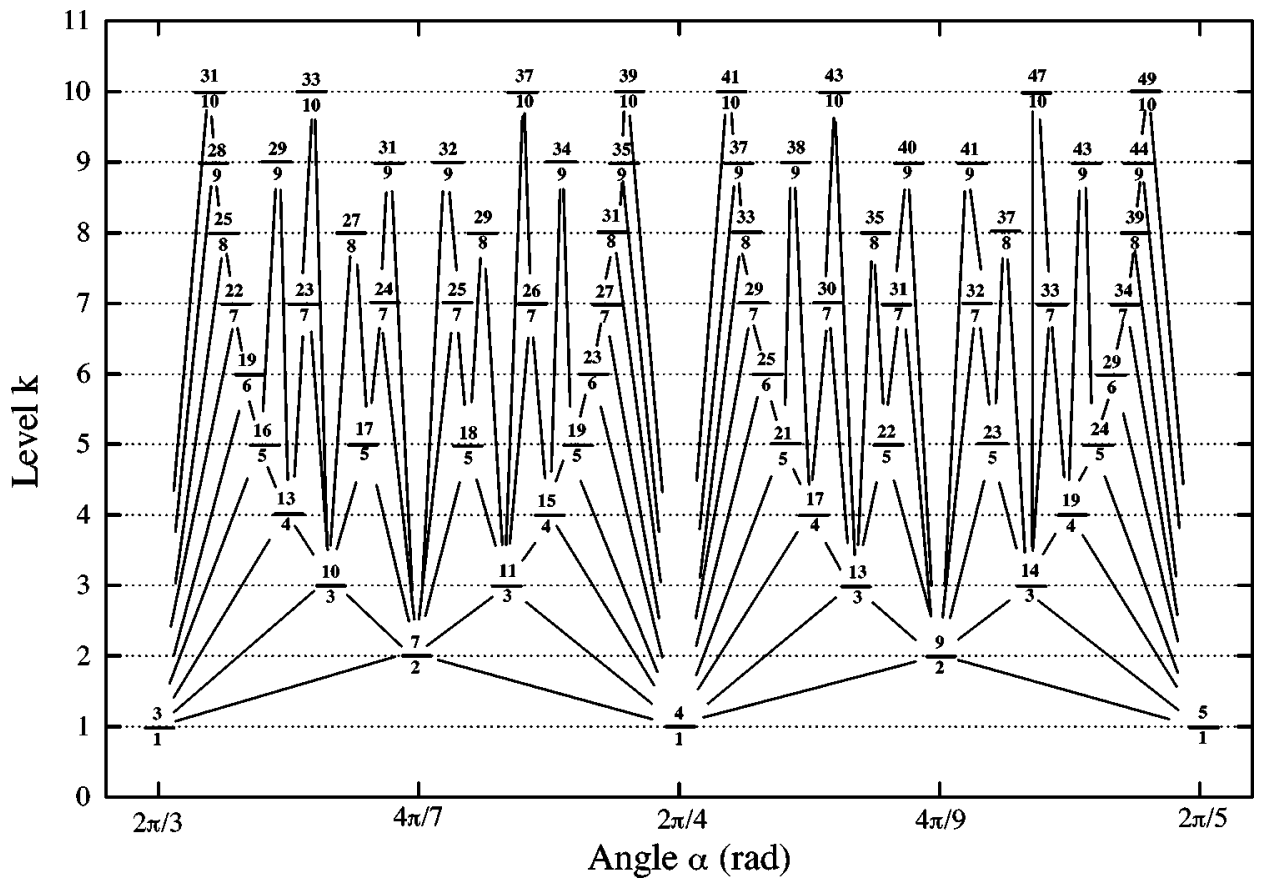

FIG. 5. Farey tree limited to angles between $2 \pi / 3$ and $2 \pi / 5$, up to level $k=10$. 


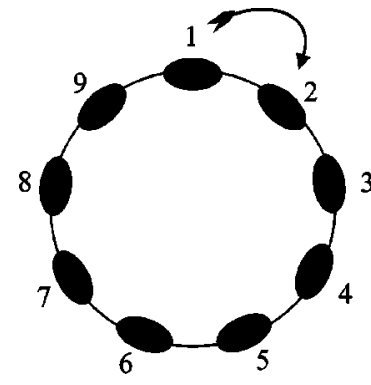

(a)

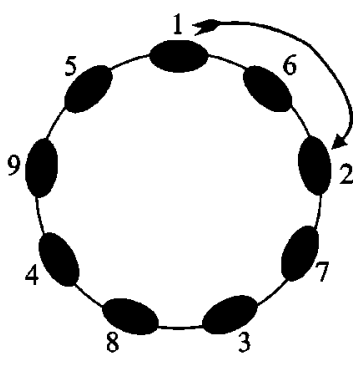

(b)
FIG. 6. Two ways of creating a nine-spot pattern: (a) by building a spot on one location after the other, corresponding to the case $k=1$ and $\alpha=1 / 9 \times 2 \pi$; (b) or by jumping over one location, meaning that the entire pattern explores the complete circle twice. This case corresponds to $k=2$ and $\alpha=2 / 9 \times 2 \pi$.

as a function of the initial intensity $X_{0}$ produced at the time $t=0$ at the position $i=1$ of the first spot, and of the persistence $P$ is as follows:

$$
X_{c, k}(t=q \tau, i)=\sum_{j=0}^{n j+i-k \leqslant q} C_{q}^{n j+i-k} P^{q-n j-i+k} X_{0} .
$$

The expression $X_{c, k}(t=q \tau, i) / X_{0}$ is illustrated in Table II in the case $c=7, k=2$. This table explains the construction process of $X_{n, k}(t=q \tau, i)$.

If the persistence $P$ depends on the intensity, then the comments given in Sec. III about nonlinear evolution of the intensity are also relevant.

What has been described until now corresponds to the cases where the spots are stationary. Nonstationary patterns have also been observed and are described in the next section.

\section{PATTERN ROTATION}

If $\alpha$ is slightly different from $2 \pi / n$, the spots rotate on the screen with a constant velocity that depends on the difference between $\alpha$ and $2 \pi / n$. This motion is explained by the fact that, after one loop, the pattern does not match the previous one exactly, but is slightly moved forward or backward, so that the system gives the visual impression of a rotation of the spots. This is very similar to what is observed when a rotating object is illuminated with a stroboscope. This rotating pattern is characterized not only by the number of spots, but also by the frequency $f=1 / T$, where $T$ is the time needed for the whole pattern to make a complete turn. By continuously changing the angle $\alpha$ from $2 \pi / n$ to $2 \pi /(n+1)$, the $n$-spot pattern begins to rotate with a frequency that increases linearly with the difference between $\alpha$ and the angle $2 \pi / n$ of the stationary pattern. After a more complex transition state, the spots reorganize into an ( $n$ $+1)$-spot pattern rotating in the opposite direction. The frequency decreases until it completely stops. This corresponds to a transition where the level $k$ remains equal to 1 . In other cases, patterns of different levels $k$ can be observed during the transition, as described in the following example.

The transition from case $9 / 2$ to case $5 / 1$ is represented in Fig. 7. A circular line intercepting all the spots was sampled every $1 / 25 \mathrm{~s}$. Then the lines are horizontally plotted one below the other. This represents a spatiotemporal diagram. The juxtaposition of these lines shows the evolution of the pattern as the angle $\alpha$ is continuously decreased from $2 / 9 \times 2 \pi$ to $1 / 5 \times 2 \pi \mathrm{rad}$ (time increases from top to bottom). Each spot appears as a white strip, and its rotation speed is given by the slope of the strip. The $9 / 2$ spots are stationary at the beginning of the transition, but they progressively rotate as the angle is decreased. They rotate with an increasing frequency. Before reaching the equilibrium state $n=5, k=1$, other different states can be observed in this figure and can be followed on the Farey tree: 24, 19, and 14 spots can be seen corresponding, respectively, to the nodes 24/5, 19/4, and 14/3. In this transition, an analogy with a superposition of a right- and a left-circular-traveling wave of different amplitudes can be made. Each one corresponds to a different quantity of spots. For instance, at the angle $\alpha^{*}$ on Fig. 7, a righttraveling wave of 19 spots and a left-traveling wave of 5 spots can be simultaneously distinguished. When the angle $\alpha$ moves away from $\alpha^{*}$, the amplitude of one of the waves increases compared to the other one, which progressively disappears. At the end of the transition, 5 spots are found rotating more and more slowly, until they completely stop. The frequency of the rotation can be expressed as a function of the different parameters of the experiment. It was measured with a photomultiplier watching a small area of the screen, and connected to a Fourier analyzer. The fast Fourier transform shows a spectrum of predominant frequency $f$ of large amplitude, and a second frequency of smaller amplitude. During the transition, the amplitude of the first frequency decreases while the amplitude of the second one in-

TABLE II. Example of the intensity $X_{c, k}(t=q \tau, i) / X_{0}$ at time $t=q \tau$ in the case $c=7$ and $k=2$. The intensity at time $t=q \tau$ is the sum of the intensities of the spot $i-1$ and the persistence of the spot $i$ at the previous loop [time $(q-1) \tau]$. The sum of the intensities of all spots at a given time $t=q \tau$ is $(P+1)^{q} X_{0}$.

\begin{tabular}{lllllllllll}
\hline \hline & \multirow{2}{*}{$t=0$} & $t=\tau$ & $t=2 \tau$ & $t=3 \tau$ & $t=4 \tau$ & $t=5 \tau$ & $t=6 \tau$ & $t=7 \tau$ & $t=8 \tau$ & $t=9 \tau$ \\
\hline$i=1$ & 1 & $P$ & $P^{2}$ & $P^{3}$ & $P^{4}$ & $P^{5}$ & $P^{6}$ & $P^{7}+1$ & $P^{8}+8 P$ & $P^{9}+36 P^{2}$ \\
$i=2$ & 0 & 0 & 0 & 0 & 1 & $5 P$ & $15 P^{2}$ & $35 P^{3}$ & $70 P^{4}$ & $126 P^{5}$ \\
$i=3$ & 0 & 1 & $2 P$ & $3 P^{2}$ & $4 P^{3}$ & $5 P^{4}$ & $6 P^{5}$ & $7 P^{6}$ & $8 P^{7}+1$ & $9 P^{8}+9 P$ \\
$i=4$ & 0 & 0 & 0 & 0 & 0 & 1 & $6 P$ & $21 P^{2}$ & $56 P^{3}$ & $126 P^{4}$ \\
$i=5$ & 0 & 0 & 1 & $3 P$ & $6 P^{2}$ & $10 P^{3}$ & $15 P^{4}$ & $21 P^{5}$ & $28 P^{6}$ & $36 P^{7}+1$ \\
$i=6$ & 0 & 0 & 0 & 0 & 0 & 0 & 1 & $7 P$ & $28 P^{2}$ & $84 P^{3}$ \\
$i=7$ & 0 & 0 & 0 & 1 & $4 P$ & $10 P^{2}$ & $20 P^{3}$ & $35 P^{4}$ & $56 P^{5}$ & $84 P^{6}$ \\
Total & $(P+1)^{0}$ & $(P+1)^{1}$ & $(P+1)^{2}$ & $(P+1)^{3}$ & $(P+1)^{4}$ & $(P+1)^{5}$ & $(P+1)^{6}$ & $(P+1)^{7}$ & $(P+1)^{8}$ & $(P+1)^{9}$ \\
\hline \hline
\end{tabular}




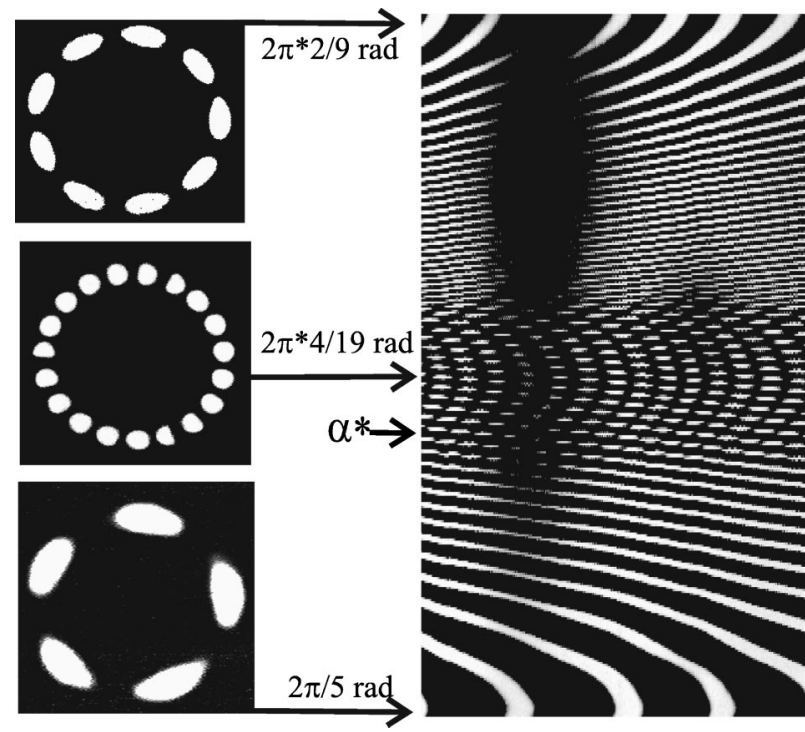

FIG. 7. Illustration of a transition between 9 and 5 spots crossing the 14-, 19-, and 24-spot symmetries. Each spot appears as a white stripe, and its rotation speed is given by the slope of the stripe. At the angle $\alpha^{*}$, a right-traveling wave of 19 spots and a left-traveling wave of 5 spots can be simultaneously distinguished. At the end of the transition, 5 spots are found rotating more and more slowly, until they completely stop.

creases and becomes predominant. On the Farey tree, for any given angle $\alpha$, each line between two nodes means that $a_{1}$ spots of the first node $a_{1} / k_{1}$ can be found rotating at a frequency $f_{1}$, or $a_{2}$ spots (node $a_{1} / k_{2}$ ) can be found rotating at another frequency $f_{2}$ in the opposite direction. The rotation frequency of any of those patterns at the angle $\alpha$, is given by $f_{\alpha}(a / k)=K(\alpha-2 \pi k / a)$ where $K=0.1352$ is a constant that depends on the experimental system. This law simply means that the rotation frequency is proportional to the distance between $\alpha$ and the angle where $a / k$ spots are stationary.

\section{ROLE OF DIFFUSION}

Diffusion in the video feedback system is the influence of a pixel on the surrounding ones. The image of a pixel has a slightly larger size than the original pixel because of defocusing. As the intensity of each pixel diffuses to its neighbors, the gray level information is mixed. The characteristic length of diffusion depends on the gap between the focused position and the defocused one where diffusion appears. In our case, diffusion plays an important part in explaining the existence of the spots. The regime associated with the appearance of spots is located exactly between $R<1$ and $R$ $>1$, where $R$ is the magnification ratio, i.e., between the behavior in which images are enlarged and the behavior where they are shrunk. The regime $R=1$ is thus unstable; the image on the screen should either grow or diminish slowly. Assuming that the magnification ratio is $R=1+\varepsilon$, a crown pattern should progressively disappear from the center of the screen to its edges. On the other hand, if $R$ is exactly equal to 1 , the image should grow with a time constant depending on the defocusing. We assume that the spots can exist only when the experimental setup is such that the disappearance of the spots at the edges of the screen is exactly compensated by diffusion.

\section{CONCLUSION}

This experiment is a model system to study spatial patterns with feedback loops, such as lasers, liquid crystals, or some hydrodynamic instabilities. The angular dependence of the number of spots is shown to follow a sequence known as the Farey tree. The patterns observed have been described and the mechanisms of their formation are given. The laws governing the intensities of the spots, such as the frequency of circular waves, have been expressed as a function of the different parameters of the system.

\section{ACKNOWLEDGMENTS}

We thank S. Fakra who started the experiments with us, M. Le Berre for her help in bibliography research, and D. Vallet for his participation in the design of the experimental setup.
[1] J. P. Gollub, T. O. Brunner, and B. G. Danly, Science 200, 48 (1978).

[2] D. Rockwell and E. Naudasher, Annu. Rev. Fluid Mech. 11, 67 (1979).

[3] S. A. Akhmanov, M. A. Vorontsov, V. Yu. Ivanov, A. V. Larichev, and N. I. Zheleznykh, J. Opt. Soc. Am. B 9, 78 (1992).

[4] F. T. Arecchi, A. V. Larichev, P. L. Ramazza, S. Residori, J. C. Ricklin, and M. A. Vorontsov, Opt. Commun. 117, 492 (1995).

[5] A. V. Larichev and F. T. Arecchi, Opt. Commun. 113, 53 (1994).

[6] M. A. Vorontsov and W. B. Miller, Self-Organization in Optical Systems and Applications in Information Technology (Springer, Berlin, 1995).

[7] E. Yao, F. Papoff, and G.-L. Oppo, Opt. Commun. 115, 73 (1998).
[8] S. Aumaitre, M. Le Berre, E. Ressayre, and A. Tallet, Quantum Semiclassic. Opt. 7, 795 (1995).

[9] D. Hennequin, D. Dangoisse, and P. Glorieux, Phys. Rev. A 42, 6966 (1990).

[10] D. Hennequin, L. Dambly, D. Dangoisse, and P. Glorieux, J. Opt. Soc. Am. B 11, 1 (1994).

[11] D. Hennequin, D. Dangoisse, and P. Glorieux, Opt. Commun. 79, 200 (1990).

[12] R. Lang and K. Kobayashi, IEEE J. Quantum Electron. QE16, 347 (1980).

[13] R. Abraham, in Structural Stability, the Theory of Catastrophes, and Applications, Vol. 525 of Lecture Notes in Mathematics, edited by I. Stewart (Springer-Verlag, New York, 1976), p. 10.

[14] G. Hausler and M. Simon, Opt. Acta 25, 327 (1978).

[15] G. Hausler and N. Stribel, Opt. Acta 30, 171 (1983).

[16] G. Hausler, G. Seckmeyer, and T. Weiss, Appl. Opt. 25, 4656 (1986). 
[17] G. Hausler and E. Lange, Appl. Opt. 29, 4798 (1990).

[18] J. P. Crutchfield, IEEE Trans. Circuits Syst. 35, 770 (1988).

[19] V. N. Goulobev, M. I. Rabinovich, V. I. Talanov, V. V. Shklover, and V. G. Yakhno, Pis'ma Zh. Éksp. Teor. Fiz. 42, 84 (1985) [JETP Lett. 42, 99 (1985)].

[20] D. L. Gonzalez and O. Piro, Phys. Rev. Lett. 55, 17 (1985).
[21] J. Maselko and H. Swinney, J. Chem. Phys. 85, 6430 (1986).

[22] J. Maselko and H. Swinney, Phys. Lett. A 119, 403 (1987).

[23] F. Mertens, R. Imbihl, and A. Mikhailov, J. Chem. Phys. 99, 8668 (1993).

[24] T. Allen, Physica D 6, 305 (1983). 